\title{
Iatrogenic 'torsade de pointes' ventricular tachycardia
}

\author{
Nicholas G. Kounis \\ M.D. \\ Chest Branch, East Birmingham Hospital, Birmingham, England
}

\begin{abstract}
Summary
Three patients who developed a distinctive form of ventricular tachycardia with oscillating QRS axis, while they were receiving drugs known to prolong the repolarization time are described. In one of the patients suffering from psychiatric illness and receiving psychotropic drugs the arrhythmia was fatal. It is postulated that the tendency to this arrhythmia was augmented by repeated electric counter shocks and negative bathmotropic drugs. Functional factors may contribute to the pathogenesis of this arrhythmia which seems to constitute an entity.
\end{abstract}

\section{Introduction}

Paroxysmal ventricular tachycardia in which the QRS axis oscillates over runs of 3 to 20 beats with definite changes in the direction has been recently recognized as the result of pharmacological overdose or side effects of certain drugs (Fazzini, Marchi and Pucci, 1973, 1975; Krikler and Curry, 1976a, b). This arrhythmia constitutes a distinctive type of ventricular tachycardia with the accepted term from the French literature of torsade de pointes ventricular tachycardia (Krikler and Curry, 1976a, b). It is caused by prolonged myocardial repolarization time manifested by prolongation of QT or QU interval (Bens et al., 1973; Fazzini et al., 1975), which favours a state of asynchronous depolarization (Han et al., 1966) and encourages re-entry processes (Raynaud et al., 1969; Evans et al., 1976).

The drugs which, so far, have been implicated to induce torsade de pointes ventricular tachycardia are cardioactive agents such as quinidine (RainierPope et al., 1962; Selzer and Wray, 1964), procainamide (McCord and Taguchi, 1951; Castellanos and Salhanick, 1967), and lignocaine (Krikler and Curry, 1976a), antianginal drugs such as prenylamine (Puritz et al., 1977) and amiodarone (Bens et al., 1973), psychotropic agents (Fowler et al., 1976), such as phenothiazines (chlorpromazine, thioridazine, trifluoperazine, mesoridazine) and tricyclic antidepressants (amitriptyline, imipramine, protriptiline, nortriptyline,) diuretics, corticosteroids and glycyrrhizin (Bens et al., 1973), which induce hypokalaemia. Torsade de pointes can complicate
(Krikler and Curry, 1976) myocardial ischaemia (Prinzmetal's variant angina, myocardial infarction), electrolytic deficits (hypokalaemia, hypomagnesaemia), acquired heart disease (myocarditis, coronary arteriosclerosis), slow basic rhythm (sino-atrial disease, high degree AV block), electrical ventricular stimulation and congenital disorders with deafness (Jervell-Lange-Nielsen syndrome, Romano-WardBarlow syndrome) with apparent (or only after exercise as a forme fruste) QT prolongation (Bernuth et al., 1973).

The following report emphasizes the iatrogenic nature of the arrhythmia which can have a fatal outcome.

\section{Case reports \\ Patient 1}

A 68-year-old man was admitted for investigations of his fainting attacks. He had suffered from chronic bronchitis for 10 years and had had a myocardial infarction 2 years before. His fainting attacks started 2 months before admission when the only medication was prenylamine lactate tablets $60 \mathrm{mg}$ thrice daily for his angina. A general practitioner who examined him during one of these episodes, prescribed slow release isoprenaline tablets $30 \mathrm{mg}$ thrice daily because of his slow heart rate following the episode. Although prenylamine had been discontinued, the patient continued to have fainting attacks (more often now, 3 to 4 times daily) while he was taking isoprenaline for the next 7 days. On admission and during a routine electrocardiogram he fainted owing to runs of torsade de pointes ventricular tachycardia (Fig. 1). The ECG revealed prolonged Q-T interval (QTc $0.75 \mathrm{~s}$ ), increased ventricular ectopic activity and prominent $U$ waves. Successive treatment with bolus lignocaine, followed by intravenous infusion, quinidine sulphate tablets $200 \mathrm{mg} 4$ times daily, and repeated (18) DC countershocks, although initially successful, seemed to worsen the condition and made the episodes more frequent. Chest X-ray, ECG electrolytes and urinary catecholamines were normal. Cardiac screening revealed systolic expansion at cardiac apex consistent with a ventricular aneurysm. Electrophysiological studies showed 


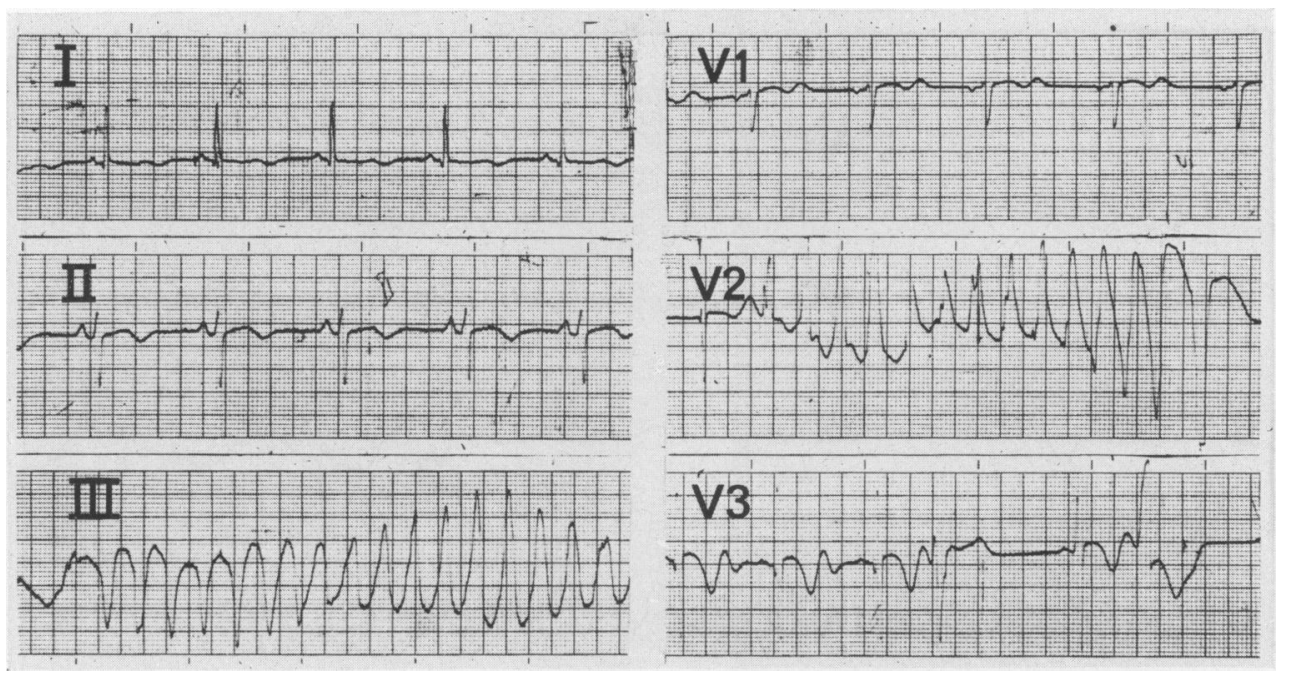

FIG. 1. Routine electrocardiogram showing torsade de pointes in leads III and $\mathrm{V}_{2}$.

prolonged repolarization time, increased ventricular ectopic activity to stimuli during the refractory period and normal SA and AV nodal studies. The arrhythmia finally was suppressed with mexiletine without any sequelae.

\section{Patient 2}

A 63-year-old woman with a 10-year history of depression was admitted to hospital after an episode of syncope. The patient was receiving thioridazine hydrochloride tablets $50 \mathrm{mg} 4$ times daily and amitriptyline hydrochloride tablets 25 $\mathrm{mg}$ thrice daily at the time of syncope. She gave a 2-year history of syncopal attacks but these were thought to be due to vertebro-basilar insufficiency. There was no past history of cardiac disease. On admission, a routine ECG (Fig. 2) revealed markedly prolonged Q-T interval (QTc 0.80 s). Clinical examination revealed no abnormality. Chest X-ray, electrolytes, cardiac enzymes, echocardiogram, plasma cortisol and peripheral blood counts were normal. Two days after admission she experienced 2 syncopal attacks accompanied by weakness, dizziness, nausea and vomiting. Electrocardiographic monitoring revealed runs of ventricular tachycardia resembling the torsade de pointes form. A bolus injection of $100 \mathrm{mg}$ of lignocaine abolished the arrhythmias, but while she was on lignocaine infusion the arrhythmia reappeared. Treatment with procainamide hydrochloride $500 \mathrm{mg} 4$ times daily did not affect the runs of torsade de pointes and it looked likely that quinidine and repeated direct current countershocks aggravated the situation. Transvenous ventricular pacing was introduced but it provoked ventricular tachycardia culminating in ventricular fibrillation which did not respond to DC countershocks and the patient died. Post-mortem examination revealed no gross or microscopic cardiac abnormality and histology of the conducting system showed normal SA and AV nodes with normal common and bifurcated bundle of His.

\section{Patient 3}

A 51-year-old man was admitted to hospital with severe retrosternal pain and a diagnosis of anteroseptal myocardial infarction was made. The patient was taking, at the time of admission, prednisolone tablets $5 \mathrm{mg}$ daily for his bronchial asthma and frusemide $\mathbf{4 0} \mathrm{mg}$ daily for congestive cardiac failure. The prednisolone tablets had been taken continuously for the last 5 years and the frusemide tablets for the last one year. Two hours after admission he developed acute left ventricular failure and he was treated with frusemide $80 \mathrm{mg}$ i.v. and venesection. He improved thereafter but the following day he developed 18 episodes of ventricular tachycardia resembling the torsade de pointes. The plasma potassium was $3 \mathrm{mmol} / \mathrm{l}$ on admission and 2.8 $\mathrm{mmol} / \mathrm{l}$ the following day during the attacks of arrhythmia. Careful examination of his ECG showed $U$ wave accentuation and QT and QU prolongation (QTc 0.78 s). Chest X-ray, echocardiogram, plasma cortisol, urinary potassium excretion and peripheral blood counts were normal. His 


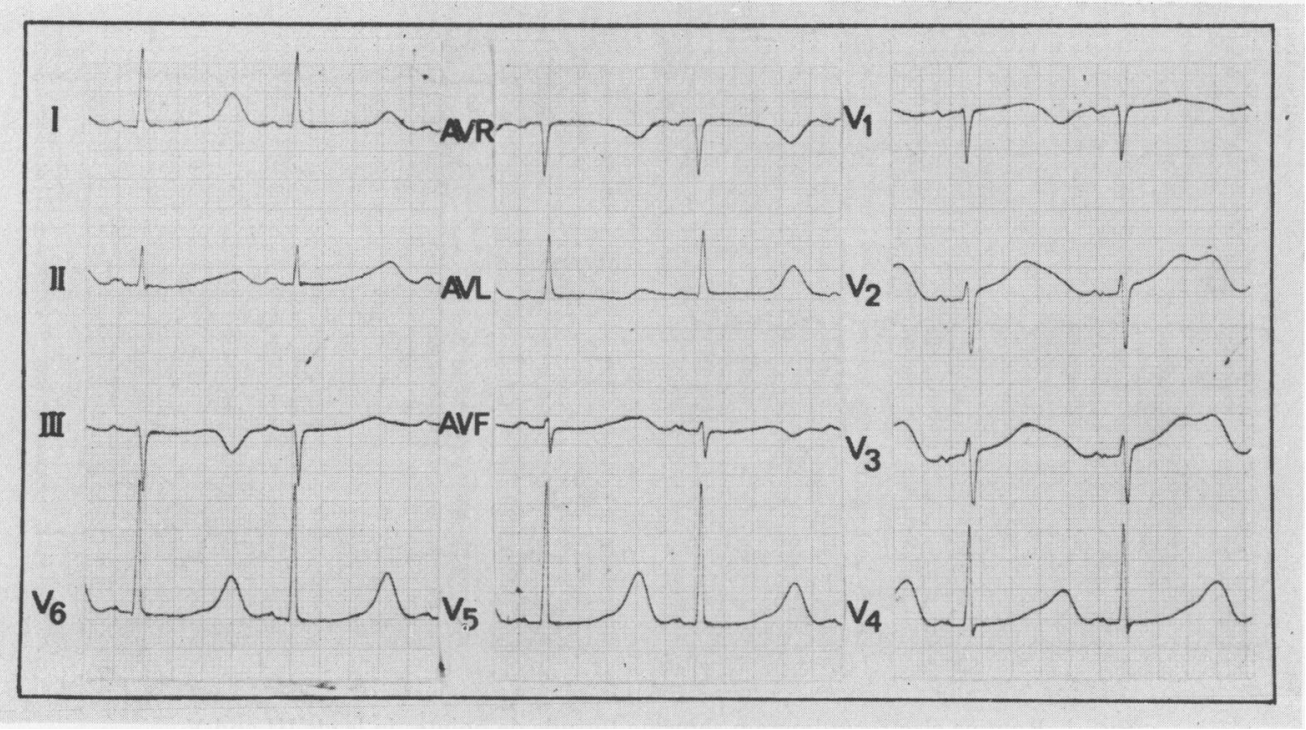

FIG. 2. Electrocardiogram showing marked prolongation of Q-T interval in patient 2.

arrhythmia was successfully treated with lignocaine and the hypokalaemia with potassium chloride infusion $(64 \mathrm{mmol}$ in $24 \mathrm{hr}$ ) and spironolactone tablets $25 \mathrm{mg} 4$ times daily. No further sequelae were noticed during his stay in hospital and after his discharge.

\section{Discussion}

The cases described developed repeated attacks of ventricular tachycardia while receiving antianginal, psychotropic, inducing hypokalaemia and negative bathomotropic agents. Two of these patients had suffered from ischaemic heart disease also. In all patients the arrhythmia seemed to be aggravated by the repeated direct current countershocks. In one of the patients histological examination of the conducting system failed to reveal any pathological changes. In this patient functional rather than anatomical factors may be implicated. This fact underlines the complexity in the pathogenesis of this arrhythmia. Ventricular arrhythmias known as quinidine syncope have long been recognized (Vico, Marvin and White, 1923; Parkinson and Campbell, 1929) in patients suffering, not only from coronary heart disease but also from pulmonary embolism and aortic and mitral valve disease. Considerable evidence suggests that prolongation of QT interval or the presence of giant $U$ waves is directly related to this type of tachycardia and in the presence of normal QT such arrhythmias do not occur (Reynolds and Vander Ark, 1976). In patients with transient hemiparesis following anaesthesia with meperidine, hydroxyzine, atropine, morphine and curare and during treatment with trifluoperazine (Reynolds and Vander Ark, 1976), ventriculag arrhythmias have appeared during the transient prolongation of QT interval, but they did noథ recur when the QT interval became normal. The long QT interval and the arrhythmias disappeared when the drug was discontinued.

Electrolytic disturbance and especially hypokalaemia increases the ectopic activity and decreases the conduction velocity in AV node (Curry et al., 1976). This presumably induced the torsade de pointes in the third patient. The presence of $U$ waves in the described patients was due to hypokalaemia, phenothiazines and negative bathomotropic drug administration. However, in hypocalcaemia, which is not associated with ventricular arrhythmias, $\mathrm{U}$ waves cannot be identified. In hypocalcaemia the ST segment is flat with delayed onset and end of $\mathrm{T}$ wave which occupies a modestly increased time interval, if at all (Reynolds and Vander Ark, 1976).

The first of the above patients continued to have torsade de pointes attacks while he was taking slow release tablets of isoprenaline hydrochloride. Isoprenaline shortens the ventricular repolarization time and thus prevents the state of asynchronous depolarization. While isoprenaline infusion shortens the QT interval (Abildskov, 1976) rapid intravenous infusion of isoprenaline may prolong it (Yanowitz, Preston and Abildskov, 1966). In this patient, isoprenaline either aggravated the situation (Kounis, 
1976), or its effect in accelerating the heart rate and shortening the QT interval had been inadequate (Krikler and Curry, 1976). Isoprenaline and atrial pacing have been shown to be of value in the treatment of torsade de pointes (Slama et al., 1973). However, a cautious trial is necessary to determine whether the rhythm disturbance is improved or worsened by isoprenaline (Puritz et al., 1977).

It looks likely that the arrhythmogenicity of the above drug is due to facilitation of re-entrant excitation as a result of decreased conduction velocity and temporal dispersion of the action potentials in different types of cardiac fibres (Arita and Surawicz, 1973).

Although the above described iatrogenic arrhythmia is not frequently encountered, knowledge of its existence should be kept in mind by physicians and precautions should always be taken.

\section{References}

AbILDSKov, J.A. (1976) Adrenergic effects on Q-T interval of the electrocardiogram. American Heart Journal, 22, 210.

ARITA, M. \& SURAwICZ, B. (1973) Electrophysiologic aspects of phenothiazines on canine cardiac fibers. Journal of Pharmacology and Experimental Therapeutics, 184, 619.

Bens, J.L., Buboisset, M., Quiret, J.C., Lesbre, J.P. \& Bernasconi, T. (1973) Syncopes par torsade de pointes induites ou favorisées par la prenylamine. Archives des maladies du roeur, des vaisseaux et du sang, 66, 1427.

Bernuth, G. von, Belz, G.G., Evertz, W. \& Stanch, M. (1973) QTU abnormalities, sinus bradycardia and AdamsStokes attacks due to ventricular tachyarrhythmia. Acta paediatrica scandinavica, 62, 675 .

Castellanos, A. \& Salhanick, L. (1967) Electrocardiographic patterns of procaine amide toxicity. American Journal of Medical Science, 253, 52.

Curry, P., FitchetT, D., Stubbs, W. \& Krikler, D. (1976) Ventricular arrhythmias and hypokalaemia. Lancet, ii, 231.

Evans, T.R., Curry, P.V.L., Fitchett, D.H. \& Krikler, D.M. (1976) "Torsade de pointes" initiated by electrical ventricular stimulation. Journal of Electrocardiology, 9, 255.

Fazzini, P.F., Marchi, F. \& Pucci, P. (1973) Q-T lungo, syncope e prenilamina. Giornale italiano di cardiologia, 3, 233.

Fazzini, P.F., Marchi, F. \& Pucci, P. (1975) Ventricular tachycardia: a new iatrogenic possibility (annotation). American Heart Journal, 90, 805.

Fowler, N.O., McCall, D., Chou, T.C., Holmes, J.C. \& HAMENSON, I.B. (1976) Electrocardiographic changes and cardiac arrythmias in patients receiving psychotropic drugs. American Journal of Cardiology, 37, 223.

HaN, J., Millet, D., Chizzonitti, B. \& Moe, G.K. (1966) Temporal dispersion of recovery of excitability in atrium and ventricle as a function of heart rate. American Heart Journal, 71, 481.

KounIs, N.G. (1976) Torsade de pointes (Letter). British Heart Journal, 39, 338.

KRIKLER, D.M. \& CURRY, P.V.L. (1976a) Torsade de pointes, an atypical ventricular tachycardia (Editorial). British Heart Journal, 38, 117.

KrIKLER, D. \& CuRrY, P.V.L. (1976b) Torsade de pointes (Letter). British Heart Journal, 39, 338.

MCCORD, M.C. \& TAGUCHI, J.T. (1951) A study of the effect of procainamide hydrochloride in supraventricular arrhythmias. Circulation, 4, 387

Parkinson, J. \& Campbell, M. (1929) Quinidine treatment of auricular fibrillation. Quarterly Journal of Medicine, 22 281.

Puritz, R., Henderson, M.A., Baker, S.N. \& Chamberlain, D.A. (1977) Ventricular arrythmias caused by prenylamine. British Medical Journal, 4, 608.

Rainier-Pope, C.R., Schrite, V., Bech, W. \& Barnard, C. (1962) The treatment of quinidine-induced ventricular fibrillation by closed-chest resuscitation and external defibrillation. American Heart Journal, 63, 582.

Raynaud, R., Brochier, M., Neel, J.L., Fauchier, J.P. \& RAYNAUD, P. (1969) Tachycardie ventriculaire à foyer variable et dyskaliémie. Archives des maladies du coeur, des vaisseaux et du sang, 62, 1578.

Reynolds, E.W. \& VANDER ARK, C.R. (1976) Quinidine syncope and the delayed repolarization syndromes. Modern Concepts in Cardiovascular Disease, 45, 117.

Selzer, A. \& WraY, H.W. (1964) Quinidine syncope. Paroxysmal ventricular fibrillation occurring during treatment of chronic atrial arrhythmias. Circulation, 30, 17.

Slama, R., Coumel, P., Motte, G., Gourgon, R., WaynBERGER, M. \& Touche, S. (1973) Tachycardies ventriculaires et torsade de pointes: frontières morphologiques entre les dysrhythmies ventriculaires. Archives des maladies du coeur, des vaisseaux et du sang, 66, 1401.

Vico, L.E., MARvin, A.M. \& WhITE, P.D. (1925) Clinical report of the use of quinidine sulfate. Archives of Internal Medicine, 31, 345.

Yanowitz, F., Preston, J.B. \& Abildskov, J.A. (1966) Functional distribution of right and left stellate innervation of the ventricles: production of neurogenic electrocardiographic changes by unilateral alteration of sympathetic tone. Circulation Research, 18, 416. 